\title{
Large scale CMB anomalies from thawing cosmic strings
}

\author{
Christophe Ringeval, ${ }^{a, b}$ Daisuke Yamauchi, ${ }^{c}$ Jun'ichi Yokoyama ${ }^{c, d, e}$ \\ and François R. Bouchet ${ }^{b}$
}
${ }^{a}$ Centre for Cosmology, Particle Physics and Phenomenology, Institute of Mathematics and Physics, Louvain University, 2 Chemin du Cyclotron, 1348 Louvain-la-Neuve, Belgium
${ }^{b}$ Institut d'Astrophysique de Paris, UMR 7095-CNRS, Université Pierre et Marie Curie, 98bis boulevard Arago, 75014 Paris, France
${ }^{c}$ Research Center for the Early Universe (RESCEU), Graduate School of Science, The Uni- versity of Tokyo, Tokyo 113-0033, Japan
${ }^{d}$ Department of Physics, Graduate School of Science, The University of Tokyo, Tokyo 113- 0033, Japan.
${ }^{e}$ Kavli Institute for the Physics and Mathematics of the Universe (Kavli IPMU), WPI, The University of Tokyo, Kashiwa, Chiba, 277-8568, Japan
E-mail: christophe.ringeval@uclouvain.be, yamauchi@resceu.s.u-tokyo.ac.jp, yokoyama@resceu.s.u-tokyo.ac.jp, bouchet@iap.fr

\begin{abstract}
Cosmic strings formed during inflation are expected to be either diluted over super-Hubble distances, i.e., invisible today, or to have crossed our past light cone very recently. We discuss the latter situation in which a few strings imprint their signature in the Cosmic Microwave Background (CMB) Anisotropies after recombination. Being almost frozen in the Hubble flow, these strings are quasi static and evade almost all of the previously derived constraints on their tension while being able to source large scale anisotropies in the CMB sky. Using a local variance estimator on thousand of numerically simulated NambuGoto all sky maps, we compute the expected signal and show that it can mimic a dipole modulation at large angular scales while being negligible at small angles. Interestingly, such a scenario generically produces one cold spot from the thawing of a cosmic string loop. Mixed with anisotropies of inflationary origin, we find that a few strings of tension $G U=\mathcal{O}(1) \times 10^{-6}$ match the amplitude of the dipole modulation reported in the Planck satellite measurements and could be at the origin of other large scale anomalies.
\end{abstract}

Keywords: Cosmic Microwave Background, Cosmic Strings, Power Asymmetry, Cold Spot 


\section{Contents}

1 Introduction $\quad 1$

2 All sky CMB maps $\quad 2$

2.1 Method 2

2.2 Nambu-Goto numerical simulations 3

2.3 Temperature maps $\quad 5$

2.4 Angular power spectrum and constraints 6

3 Induced power asymmetry $\quad 9$

3.1 Dipole in local variance maps $\quad 9$

3.2 Averaging angle dependency 12

3.3 Mixture of string-induced and Gaussian anisotropies 12

3.3.1 Mean values 12

3.3.2 Local variance maps 14

$\begin{array}{ll}3.4 \text { Discussion } & 14\end{array}$

4 Cold spots from thawing loops 15

5 Conclusion

\section{Introduction}

Although cosmic strings are a natural outcome of the symmetry breaking mechanism in the early Universe [1, 2], or may be more fundamental objects from String Theory [3, 4], they have escaped all dedicated searches in astronomical and cosmological observables to date (see Refs. [5-11] for reviews). Being active and non-Gaussian sources of CMB anisotropies [1215], current measurements by the Planck satellite allow strings to contribute no more than a few percent to the overall power spectrum and bispectrum. This translates into an $95 \%$ confidence upper bound on the allowed string tension: $G U \leq 3.2 \times 10^{-7}$ for Abelian Higgs string and $G U<1.5 \times 10^{-7}$ for Nambu-Goto strings [16-20]. It is important to notice that these results rely on the assumption that a cosmic string network evolves according to the so-called scaling regime. Indeed, in an expanding Universe, it has been shown that after a few e-folds of decelerated expansion the distribution of cosmic strings reaches an attractor in which the network's statistical properties become an universal function of the Hubble radius only [21-24]. Typically, the scaling regime for Nambu-Goto strings corresponds, at any times, to ten long strings crossing the observable Universe complemented with a power law distribution of smaller loops [25-29]. Assuming "scaling" is usually well motivated for observational purposes. If strings are formed around the Grand Unified Theory (GUT) energy scales, there is indeed plenty of time for any initial configuration to relax towards the attractor.

Within our current understanding of the early Universe, there are various explanations for strings to remain elusive in the cosmological measurements. The simplest is that their tension is relatively small compared to GUT scale, typically lower than $\left(10^{15} \mathrm{GeV}\right)^{2}$, and they remain undetectable for the moment. Another explanation is that, as for monopoles, 
cosmic strings may have been diluted away by cosmic inflation, provided they have been formed before inflation. Or, they may have never been formed at all. Both explanations would however require some fine tuning within the symmetry breaking schemes at work in the cooling of the fundamental interactions [30, 31].

Here we discuss another proposal which is that cosmic strings may have been formed during inflation [32-42]. As discussed in Ref. [43], the network statistical properties are no longer universal as they depend on when and how the strings have been formed during inflation. If they have been formed more than, say, 60 e-folds before the end of inflation (the precise number is inflation- and reheating-dependent), strings would be diluted enough not to be observable at all. If, on the contrary, they have been formed closer to the end of inflation, the typical correlation length of the string network would remain super-Hubble for most of the radiation and matter eras of the Universe, but will eventually enter the Hubble radius at some point. Following Ref. [43], we refer to this scenario as delayed scaling. As shown in this reference, delayed scaling strings induce less CMB anisotropies than a scaling network because less strings intercept our past light cone. In particular, the reduction in power preferentially occurs on the smallest length scales (large multipoles). As a result, these scenarios naturally imprint the CMB anisotropies on large scales only and one may wonder whether they could be a viable explanation of the large scales anomalies reported in the Wilkinson Microwave Anisotropies Probe and Planck measurements [44-48].

In the following, we focus on a delayed scaling scenario in which the strings cross our past light cone after recombination. In order to derive their observable imprints, we use numerical simulations of Nambu-Goto strings in which we propagate photons to ray-trace the CMB sky using a method similar to the one of Ref. [49] and described in section 2. In particular, we find that starting with a Vachaspati-Vilenkin string network [50] having a super-Hubble correlation length at last scattering, $\xi \simeq 32 \eta_{\text {lss }}$ (in comoving coordinates, $\eta$ being the conformal time) our past light cone would contain from zero to two strings which remain almost static till today. The resulting CMB angular power spectrum ends up being at least two orders of magnitude lower than the one produced by a scaling network such that the scenario ends up being constrained only by direct searches of rare Gott-Kaiser-Stebbins [51, 52] (GKS) temperature discontinuities [53-57]. As discussed below, this typically allows string tension up to $G U=\mathcal{O}(1) \times 10^{-6}$ to be compatible with current constraints. In section 3 , we quantify to which extent these strings can generate a power asymmetry in the CMB sky by using a local variance estimator introduced in Ref. [58]. From a thousand random realizations of the string network with $\xi \simeq 32 \eta_{\mathrm{lss}}$, we find that the induced CMB patterns can, among other effects, generically generate a large scale dipole modulation in the local variance. We also show that the amplitude of the modulation is very peculiar to this scenario and we discuss how it is modified by changing the initial value of $\xi$ in section 4 . In particular, for an initial correlation length $\xi \simeq 16 \eta_{\text {lss }}$, our light cone can contain a thawing cosmic string loop which genuinely generates a cold spot in the CMB sky. Finally, it is found that string tensions around $G U=\mathcal{O}(1) \times 10^{-6}$ are found to mimic the currently observed dipole modulation in the Planck data [44, 58].

\section{All sky CMB maps}

\subsection{Method}

In order to derive the CMB temperature anisotropies generated by cosmic strings formed during inflation, we have performed numerical simulations of Nambu-Goto strings evolution 
in Friedmann-Lemaitre-Robertson-Walker (FLRW) spacetimes. The code used is based on an improved version of the Bennett and Bouchet Nambu-Goto cosmic string code [24]. For our purpose, the initial conditions have been set using the Vachaspati-Vilenkin algorithm [50] which attributes a random phase (correlated over a distance $\xi$ ) to an hypothetical $U(1)$ Higgs field on a three-dimensional cubic lattice. Depending on the phase topology around each corners of the cubic grid, the algorithm determines if a string actually passes, or not, through each of the cubic faces. Because the strings are assumed to be formed during inflation, we have set vanishing initial velocity to each of the strings and loops thus generated. The fundamental difference compared to the more ordinary situation in which one evolves a string network in scaling is that we now set the initial correlation length of Higgs phases to be much larger than the Hubble radius. In order to simulate the actual physical configuration, it would be necessary to set the correlation length during inflation and solve the NambuGoto dynamics for about 60 e-folds of inflation and as much e-folds of decelerated expansion during the subsequent reheating, radiation and matter eras. Of course, this is not doable numerically but it is not a limitation as the Nambu-Goto dynamics becomes trivial in the super-Hubble limit $\xi / \eta \rightarrow \infty$ ( $\eta$ being the conformal time). Indeed, strings remain frozen due to Hubble damping [59] and the overall configuration of the network is straightforwardly redshifted [43]. Therefore, we have chosen to start the simulation in the matter era at a redshift close to the last scattering surface $z=z_{\mathrm{lss}}=1089$. Our initial configuration is described by $\xi /\left.\eta\right|_{\text {lss }}$, which is an observable model parameter for the delayed scaling scenario, and gives the correlation length of the network at the time of last scattering. From the above discussion, such an approach is valid only if $\xi>\eta_{\mathrm{lss}}$ to prevent any significant difference in the network evolution before the last scattering surface with respect to simple redshifting. The resulting CMB anisotropies are completely sourced by the strings after recombination and can be computed from the integrated Sachs-Wolfe effect associated with the Nambu-Goto stress tensor of the whole network. In the transverse temporal gauge, up to a dipole term, the relative photon temperature shifts in the direction $\hat{\boldsymbol{n}}$ are given by [60-62].

$$
\frac{\Delta T}{T}(\hat{\boldsymbol{n}})=-4 G U \int_{\boldsymbol{X} \cap \boldsymbol{x}_{\gamma}} \boldsymbol{u} \cdot \frac{X \hat{\boldsymbol{n}}-\boldsymbol{X}}{(X \hat{\boldsymbol{n}}-\boldsymbol{X})^{2}} \epsilon \mathrm{d} \sigma,
$$

where the integral is over all string position vectors $\boldsymbol{X}=\left\{X^{i}\right\}$ crossing our past line cone. Here $\mathrm{d} l=\epsilon \mathrm{d} \sigma$ is the invariant string length element and $\boldsymbol{u}$ the sourcing vector of the GottKaiser-Stebbins effect (in the temporal gauge):

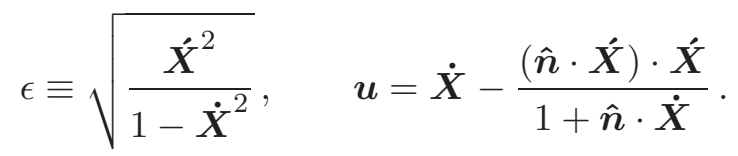

Both $\dot{\boldsymbol{X}}=\partial \boldsymbol{X} / \partial \eta$ and $\dot{\boldsymbol{X}}=\partial \boldsymbol{X} / \partial \sigma$ are extracted from the numerical simulations while the set of string position vectors $\boldsymbol{X}$ intercepting our past light cone is determined by propagating photons within the simulation box (see Ref. [49] for more details).

\section{$2.2 \quad$ Nambu-Goto numerical simulations}

In practice, our runs are performed in a unit comoving box in which we are free to set two dimensionless numerical parameters (normalized by the comoving size of the numerical box): the initial conformal time (or conformal horizon), $d_{\mathrm{h}_{\mathrm{ini}}}$ and the initial correlation length $\ell_{\mathrm{c}}$. In order to implement the delayed scaling string scenario described before, one must have: 


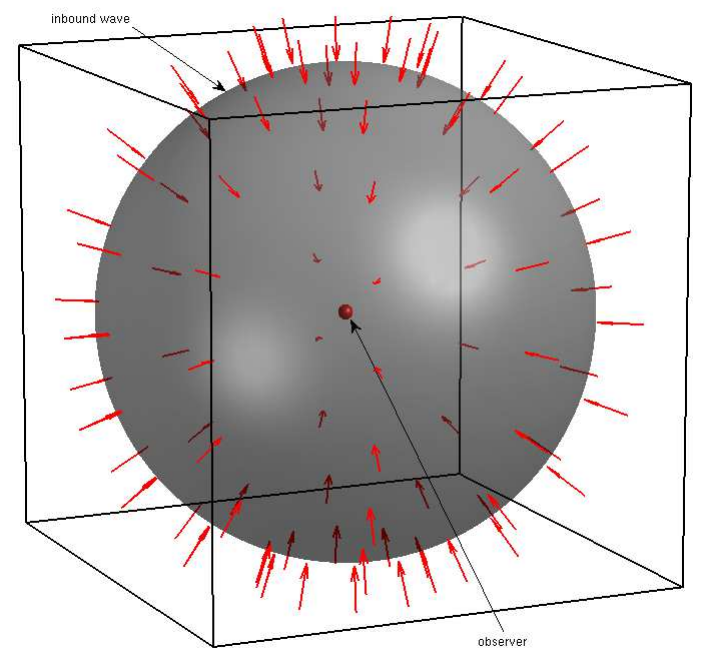

Figure 1. Sketch of the numerical simulation comoving box. An inbound spherical light-like wave is propagated towards the centre during the run to determine the string position vectors $\boldsymbol{X}$ intercepting our past light cone.

$\ell_{\mathrm{c}} / d_{\mathrm{h}_{\mathrm{ini}}}=\xi / \eta_{\mathrm{lss}}>1$. Therefore, compared to the method described in Ref. [49], which was dedicated to a scaling network having initially $\ell_{\mathrm{c}} /\left.d_{\mathrm{h}_{\text {ini }}}\right|_{\text {scal }}<1$, our simulation box contains only a very few number of strings. This renders possible to actually simulate at once the whole observable universe from the last scattering surface to today. At the same time we evolve the string network according the the Nambu-Goto equations in FLRW background, an inbound spherical wave of photons is propagated through the simulation. By recording all string segments intercepting this wave, one can reconstruct the past light cone of a virtual observer located in the centre of the sphere (see figure 1). Performing the integral of Eq. (2.1) over all directions $\hat{\boldsymbol{n}}$ gives the string induced CMB sky the observer would see. However, this also limits the time during which the simulation can be run: an inbound light-like wave initially inscribed in the unit comoving box collapses onto the observer at the numerical conformal time $d_{\mathrm{h}_{0}}=d_{\mathrm{h}_{\text {ini }}}+0.5$. Since we want to compute the network evolution over a redshift range going up to the last scattering surface, $z_{\mathrm{lss}}=1089$, this imposes $d_{\mathrm{h}_{0}} / d_{\mathrm{h}_{\text {ini }}} \simeq \sqrt{1+z_{\mathrm{lss}}}$. Solving for $d_{\mathrm{h}_{\text {ini }}}$ one gets

$$
d_{\mathrm{h}_{\mathrm{ini}}} \simeq \frac{1}{2\left(\sqrt{1+z_{\mathrm{lss}}}-1\right)} \simeq 1.56 \times 10^{-2}
$$

a value that determines the real comoving size of the numerical box ${ }^{1}$ (see Refs. [49, 54])

$$
L_{\mathrm{sim}} \simeq \frac{2}{d_{\mathrm{h}_{\mathrm{ini}}} H_{0}} \frac{1-\sqrt{\Omega_{\mathrm{rad}} / \Omega_{\mathrm{mat}}} \sqrt{1+z_{\mathrm{lss}}}}{\sqrt{\Omega_{\mathrm{mat}}} \sqrt{1+z_{\mathrm{lss}}}} \mathrm{Gpc} \simeq 20.6 \mathrm{Gpc} .
$$

$H_{0}$ stands for the Hubble parameter today and $\Omega_{\text {rad }}$ and $\Omega_{\text {mat }}$ are the density parameters today of radiation and matter.

\footnotetext{
${ }^{1}$ This is an analytic approximation assuming no cosmological constant and $\Omega_{\text {rad }} \ll \Omega_{\text {mat }}$. The actual value used to determine the angular size of string generated CMB patterns is computed exactly within the $\Lambda$ CDM model.
} 
The only remaining free parameter is $\ell_{\mathrm{c}}$. Because the initial conditions are set according to the Vachaspati-Vilenkin algorithm, namely over a comoving grid of the simulation box, $\ell_{\mathrm{c}}=1 / N_{\mathrm{c}}$, where $N_{\mathrm{c}}>1$ is an integer. Our simulations can therefore be used to evolve an initial configuration having

$$
\left.\frac{\xi}{\eta}\right|_{\mathrm{lss}}=\frac{1}{N_{\mathrm{c}} d_{\mathrm{h}_{\mathrm{ini}}}} \simeq \frac{64}{N_{\mathrm{c}}},
$$

the maximal value being obtained for $N_{\mathrm{c}}=2$, i.e., $\xi /\left.\eta\right|_{\text {lss }} \simeq 32$. One may compare this number to the ones used in Ref. [43] in which a network of super-Hubble strings was considered at an initial redshift of $z_{\mathrm{rad}}=2.3 \times 10^{7}$. With a mixture of matter and radiation, one gets

$$
\left.\left.\frac{\xi}{\eta}\right|_{\mathrm{rad}} \simeq \frac{\sqrt{1+\frac{1+z_{\mathrm{eq}}}{1+z_{\mathrm{lss}}}}-1}{\sqrt{1+\frac{1+z_{\mathrm{eq}}}{1+z_{\mathrm{rad}}}}-1} \frac{\xi}{\eta}\right|_{\mathrm{lss}} \simeq 5 \times 10^{5} .
$$

Our study is therefore probing a regime for which strings are formed relatively earlier during inflation than the cases discussed in Ref. [43]. As such they spend most of their evolution in a frozen super-Hubble configuration during which the network length scale gradually approaches the Hubble scale. At the time these two scales are matching, strings start to decouple from the Hubble flow and move under their tension. We will be referring to this regime as "thawing" because only after the correlation length falls well below the Hubble radius, the system will enter the delayed scaling regime studied in Ref. [43], and ultimately the usual scaling regime. In our treatment, only the thawing strings can be seen in the sky and contribute to the CMB anisotropies. CMB signatures on small scales disappear almost completely for such large values of the correlation length but one may still expect some CMB distortions to be present on the largest length scales. This is quantified in the following sections.

\subsection{Temperature maps}

The CMB temperature anisotropies generated by the strings are obtained by the method described in section 2.1. In order to match the Planck angular resolution, the sky has been pixelized over more than 50000000 directions $\left(N_{\text {side }}=2048\right)$ using the HEALPix library [63]. Moreover, in order to discuss the statistical properties and cosmic variance effects, we have generated 1024 realizations of these maps by running numerical simulations of Nambu-Goto strings starting from independent random realizations of the initial conditions, and random positions of the observer within the simulation volume. This is particularly relevant as the statistics of the CMB anisotropies sourced by cosmic strings is genuinely non-Gaussian [11]. As discussed below, a few strings affecting the largest CMB angular scales can significantly boost cosmic variance effects thereby changing the probability of rare events compared to what one may expect from purely Gaussian anisotropies.

In figure 2, we have represented six different realizations of the CMB temperature anisotropies, all having an initial value of $N_{\mathrm{c}}=2$, i.e., $\xi /\left.\eta\right|_{\text {lss }} \simeq 32$. As can be seen on the upper right panel, in some realizations, no string actually crosses our past light cone whereas in other situations one or two strings are visible, either completely (as in the top left panel) or some part of it (middle left). 

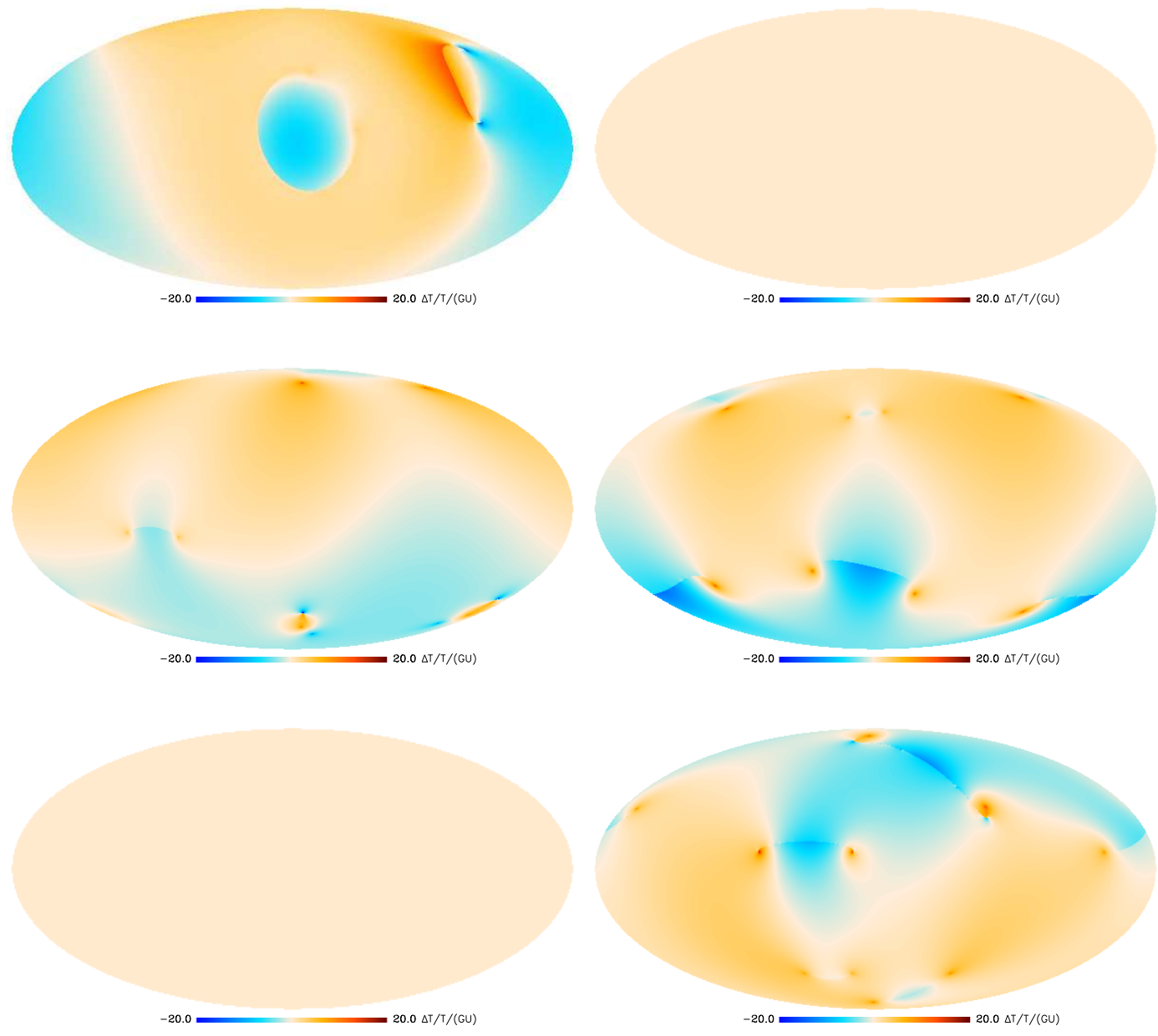

Figure 2. Six realizations of the CMB sky temperature generated by thawing scaling strings having an initial correlation length $\xi=32 \eta_{\mathrm{lss}}$ at the last scattering surface $\left(N_{\mathrm{c}}=2\right)$. In the second and fourth map (from top left to bottom right), the signal vanishes because no string crossed our past light cone. In the other panels, the temperature patterns are generated by a few loops (see first map) entering the Hubble radius and starting to evolve according to the Nambu-Goto dynamics. Sometimes, part of it intercept the last scattering surface which is why some temperature patterns seem to be attached on it. We have generated a thousand of such maps to compute their statistical properties.

\subsection{Angular power spectrum and constraints}

As above-mentioned, for $N_{\mathrm{c}}=2$, the strings have barely the time to start evolving after entering the Hubble radius and are quasi-static. Therefore, the induced CMB temperature anisotropies are orders of magnitude smaller than the ones generated by a scaling network [17]. In fact, for quasi-static strings, the induced temperature anisotropies are essentially sourced by the curvature term of $\boldsymbol{u}$ in equation (2.2). In figure 3, we have represented the mean angular power spectrum, and its standard deviation, obtained over the 1024 generated CMB maps. This figure also shows the angular power spectrum obtained from Nambu-Goto strings in scaling, as obtained from the Unequal Time Correlator (UETC) method in Ref. [19]. We have also represented the Integrated Sachs-Wolfe (ISW) contribution of scaling strings as 


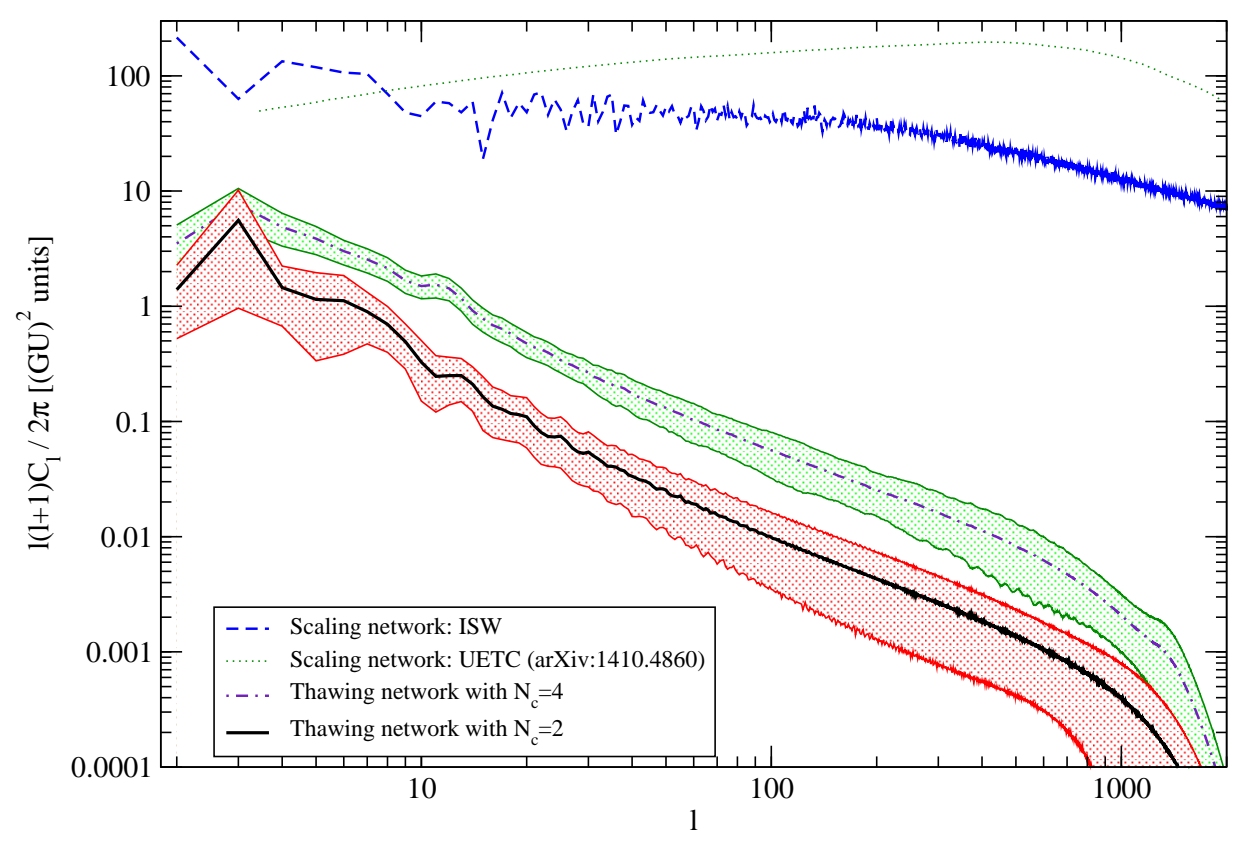

Figure 3. Mean and standard deviation of the CMB temperature angular power spectrum for the delayed scaling string network in the thawing regime with $N_{\mathrm{c}}=2$ and $N_{\mathrm{c}}=4$ (convolved with a Gaussian beam of $10^{\prime}$ ). For comparison, we have represented the total angular power spectrum for a Nambu-Goto network in scaling obtained using the UETC method (extracted from Ref. [19]) as well as the ISW contribution obtained from ray tracing [49]. Thawing strings with $N_{\mathrm{c}}=2$ have an angular power spectrum at least two orders of magnitude smaller than scaling strings.

obtained from the ray-tracing method of Ref. [49]. The ratio between the spectra of thawing strings and scaling strings at $\ell=10$ is of the order $C_{10}^{\text {thaw }} / C_{10}^{\text {scal }} \simeq 4 \times 10^{-3}$ while around the maximum power for scaling strings, at $\ell=400, C_{400}^{\text {thaw }} / C_{400}^{\text {scal }} \simeq 2.5 \times 10^{-5}$. The two-sigma upper bound on the scaling string tension obtained with the Planck data is of the order $G U \simeq 1.5 \times 10^{-7}[17,19]$, mostly coming from the angular scales around $\ell=400$ where cosmic variance effects are minimal. From $C_{\ell} \propto(G U)^{2}$, one deduces that delayed scaling strings in the thawing regime with $G U=\mathcal{O}(1) \times 10^{-6}$ remain invisible in the angular power spectrum. One may compare the resultant power spectra to those obtained in Ref. [43], in which the evolution of the delayed scaling string network was assumed to follow the velocitydependent one-scale model. The amplitude of the power spectrum turns out to be several tens of times larger than the one obtained here in our simulations. This discrepancy is mainly due to the different regimes probed. The analytic model cannot be extrapolated to the thawing regime because strings have not yet interacted enough to correlate their velocity and lengths scales. Moreover, when strings are almost static, the GKS temperature anisotropy [51, 52] becomes dominated by curvature and point-like sources such as kinks, cusps, and string end-points on the last scattering surface. These contributions are responsible for a slope at large multipoles slightly steeper than the one predicted by the usual line discontinuities (see figure 3$)$.

However, as can be seen in figure 2 , for $G U=\mathcal{O}(1) \times 10^{-6}$, the CMB sky would contain one or two string-induced temperature discontinuities having an amplitude of tens of $\mu \mathrm{K}$ that could be detectable with direct searches [57,64]. More precisely, Ref. [57] reports that string 

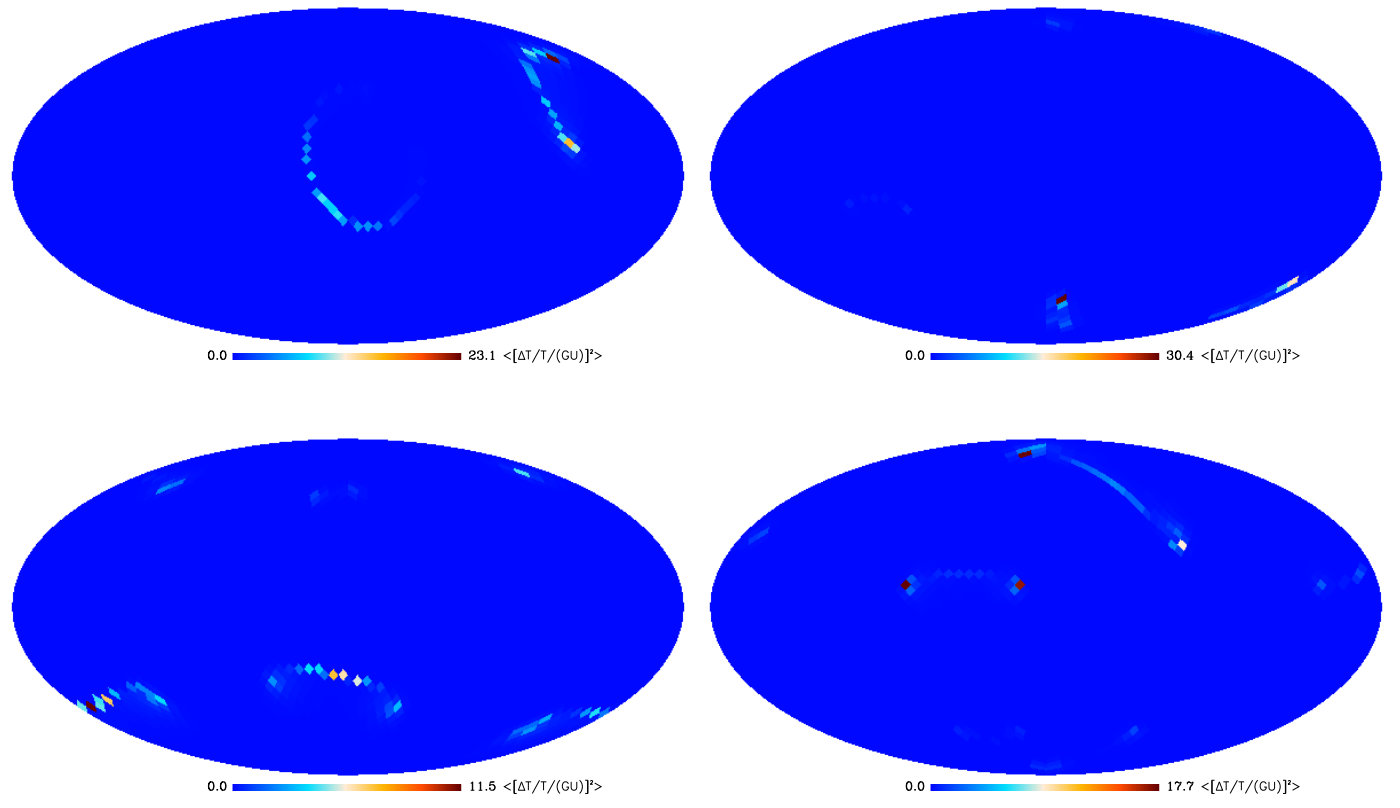

Figure 4. Local variance maps of $\left\langle[\Delta T /(T G U)]^{2}\right\rangle$ over $1.7^{\circ}$ circles associated with the four nonvanishing temperature maps of figure 2. The maximal value of the standard deviation $\sigma$ is reached along one or two directions only and equals 4.8, 5.5, 3.4 and 4.2 for the four maps, respectively.

induced discontinuities of amplitude $\Delta=117 \mu \mathrm{K}$ over patches of $1.7^{\circ}$ would be detectable in the Planck data at $95 \%$ of confidence. Notice that this result is not directly applicable to our situation as the searches performed in Refs. [57, 64] are made for scaling-like strings, namely it is assumed that there are a few strings moving at relativistic velocities in every $1.7^{\circ}$ patches in the sky. Here, the strings are almost static and there are only one or two temperature steps over the whole sky.

In order to estimate what is the amplitude of the temperature steps produced over a given angular scale in the thawing regime, we have represented in figure 4 four local variance maps computed over patches of angular opening $1.7^{\circ}$. Each of them is associated with the four non-vanishing CMB temperature maps of figure 2, i.e., corresponds to a network having $N_{\mathrm{c}}=2$. Practically, we have discretized the sky using the HEALPix pixelization scheme with $N_{\text {side }}=16$, which corresponds to 3072 directions. In each of these directions, the variance $\sigma^{2}=\left\langle[\Delta T /(T G U)]^{2}\right\rangle$ is computed over a circle having an angular opening of $1.7^{\circ}$. If no temperature step is present in that circle, $\sigma^{2}$ remains very small whereas if a string crosses the patch, $\sigma^{2}$ is proportional to $\Delta^{2}$. The maximal variance being obtained for a string cutting the circle in two equal parts [57], one has

$$
\Delta=\max (2 \sigma T G U) .
$$

Averaged over 1024 of such local variance maps, we find $\langle\Delta\rangle \simeq 7.5 T G U$ and blindly using the maximal amplitude $\Delta=117 \mu \mathrm{K}$ gives $G U \simeq 6 \times 10^{-6}$. As mentioned above, this value of $\Delta$ is certainly underestimated for the thawing strings. Indeed, instead of having one temperature step of this amplitude over each $1.7^{\circ}$ circle, the local variance maps essentially show that the delayed scaling network in the thawing regime produces only one step of amplitude $8 T G U$ 

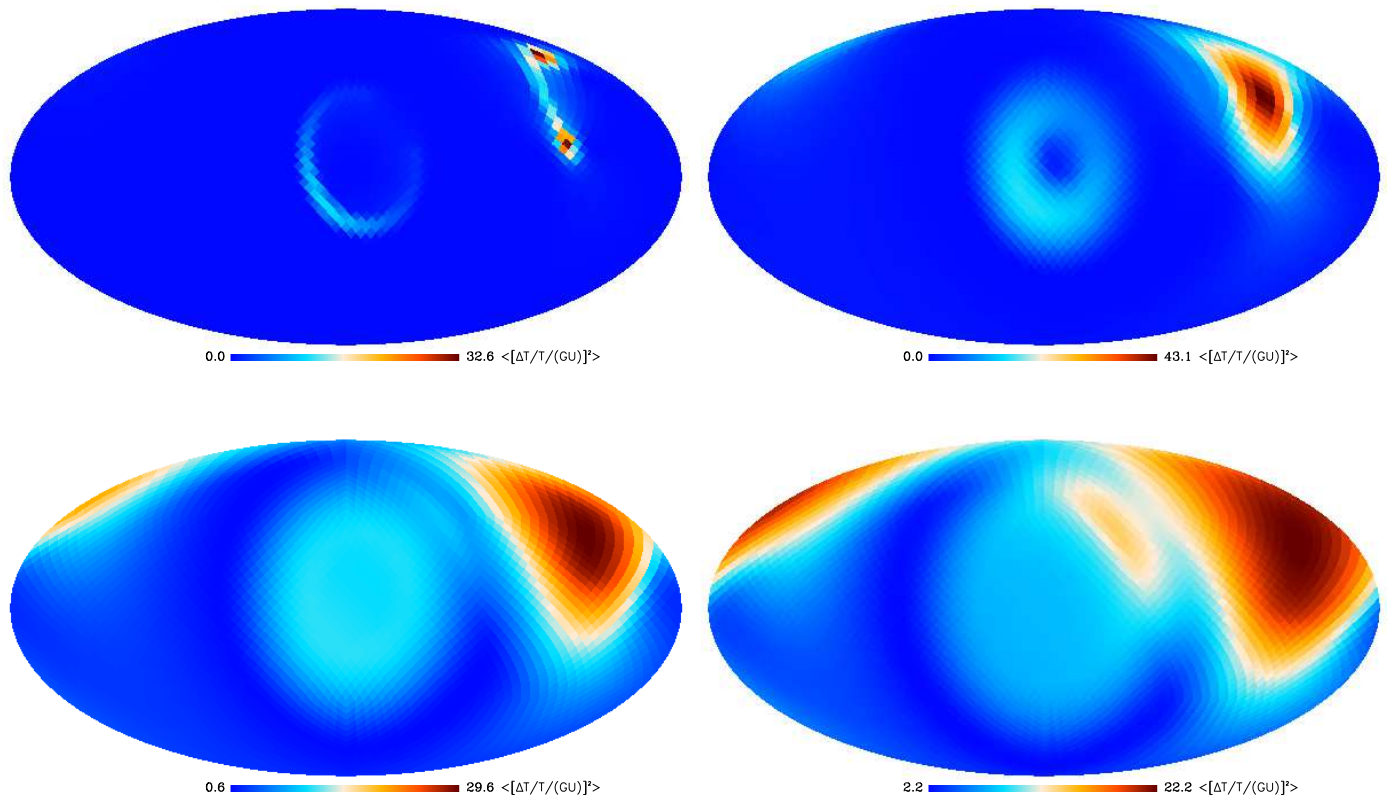

Figure 5. Local variance maps obtained from the first CMB maps of figure 2 (top left). From top left to bottom right, the averaging angles shown are $6^{\circ}, 24^{\circ}, 48^{\circ}$ and $64^{\circ}$, respectively.

over the whole sky. Nevertheless, it is certainly fair to claim that values of $G U=\mathcal{O}(1) \times 10^{-6}$ may be considered as a minimum threshold above which the string effects could be directly detectable in the CMB maps.

\section{$3 \quad$ Induced power asymmetry}

Although thawing strings let relatively weak CMB imprints at small angular scales, they genuinely generate a power asymmetry on the largest angles. As can be seen in figure 2, all maps are associated with smooth gradients over the whole sky. As reported in Refs. [44, 48], various large scale anomalies measured in the Planck 2013 and 2015 data can be phenomenologically described by a dipole modulation model. Such a model has however been shown to have some significance on the largest angular scales only [47] and, in the following, we quantify as much thawing strings could contribute to this effect.

\subsection{Dipole in local variance maps}

As discussed in Ref. [58], local variance maps of temperature anisotropies over circles of given angular size can be used to quantify the amplitude of a power asymmetry. In particular, a pure dipole modulation along a given direction $\hat{\boldsymbol{\omega}}[58,65]$

$$
\frac{\Delta T}{T}(\hat{\boldsymbol{n}})=\left.(1+A \hat{\boldsymbol{n}} \cdot \hat{\boldsymbol{\omega}}) \frac{\Delta T}{T}\right|_{\Lambda \mathrm{CDM}},
$$

would also show up in the local variance map as a dipole of amplitude $2 A \sigma_{\Lambda \mathrm{CDM}}^{2}$ :

$$
\sigma^{2}=\sigma_{\Lambda \mathrm{CDM}}^{2}+2 A \sigma_{\Lambda \mathrm{CDM}}^{2} \hat{\boldsymbol{n}} \cdot \hat{\boldsymbol{\omega}}+\mathcal{O}\left(A^{2}\right) .
$$



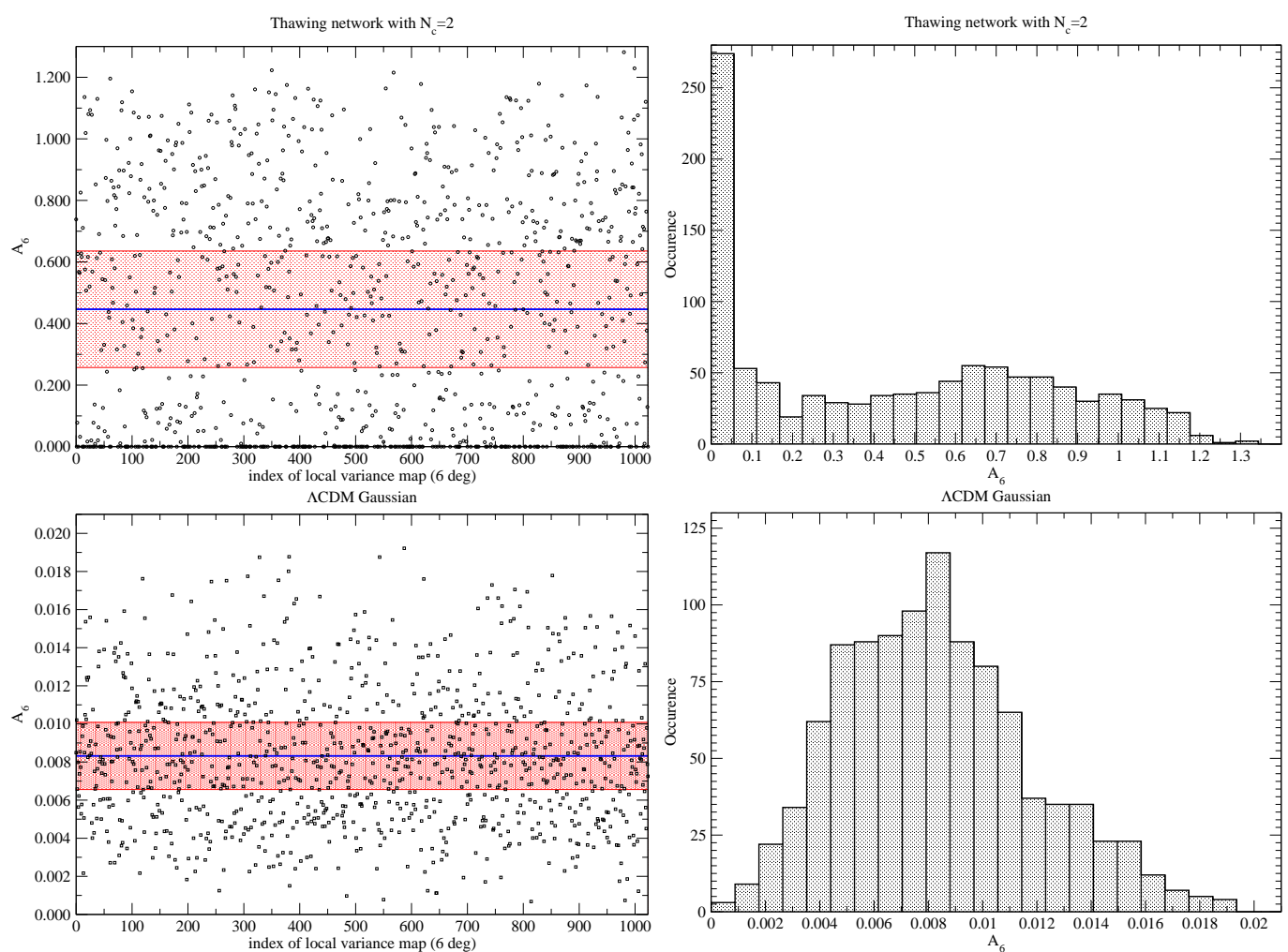

Figure 6. The values of $A_{6}=\sigma_{\text {dip }}^{2} /\left(2 \sigma_{\text {mon }}^{2}\right)$ obtained for 1024 local variance maps of angular opening $6^{\circ}$ (left) and its distribution (right). The upper panels are for the thawing strings scenario with $N_{\mathrm{c}}=2$ while the lower panels have been derived from pure $\Lambda$ CDM CMB maps. The horizontal line corresponds to the mean value over 1024 realizations while the shaded region represents the standard deviation. The dipole modulation for thawing strings can be two orders of magnitude larger than the one expected for Gaussian $\Lambda$ CDM.

As a result, one may test the existence of this anisotropy by fitting both a monopole $\sigma_{\text {mon }}^{2}$ and a dipole amplitude $\sigma_{\text {dip }}^{2}$ to the local variance maps. For a pure dipole modulation as in Eq. (3.1), the ratio $\sigma_{\text {dip }}^{2} /\left(2 \sigma_{\text {mon }}^{2}\right) \simeq A$ independently of the circle angular size. For each of the $1024 \mathrm{CMB}$ maps generated in section 2 , we have constructed as many local variance maps by averaging over circles of different angular size, namely $1.7^{\circ}, 6^{\circ}, 12^{\circ}, 24^{\circ}, 36^{\circ}, 48^{\circ}$ and $64^{\circ}$.

Figure 5 illustrates the procedure by showing the local variance maps associated with the top left realization of figure 2 and for four angles. The local variance map contains only a strong signal if a string is present within the averaging angle. Therefore, for small angles, most of the sky directions contain no strings and the local variance map exhibits a strong signal only around the strings location. On the contrary, for larger angles, many more directions in the sky may contain a string within the circle such that the string signal affects larger patches.

A monopole and a dipole are then fitted to each of these maps. The direction of the dipole is random as it changes with each realization. However, the ratio $A_{\theta}=\sigma_{\text {dip }}^{2} /\left(2 \sigma_{\text {mon }}^{2}\right)$ tells us how much the strings may generate a signal looking like a dipole modulation at a given angle $\theta$. In figure 6 , we have plotted the distribution of this ratio over 1024 local 

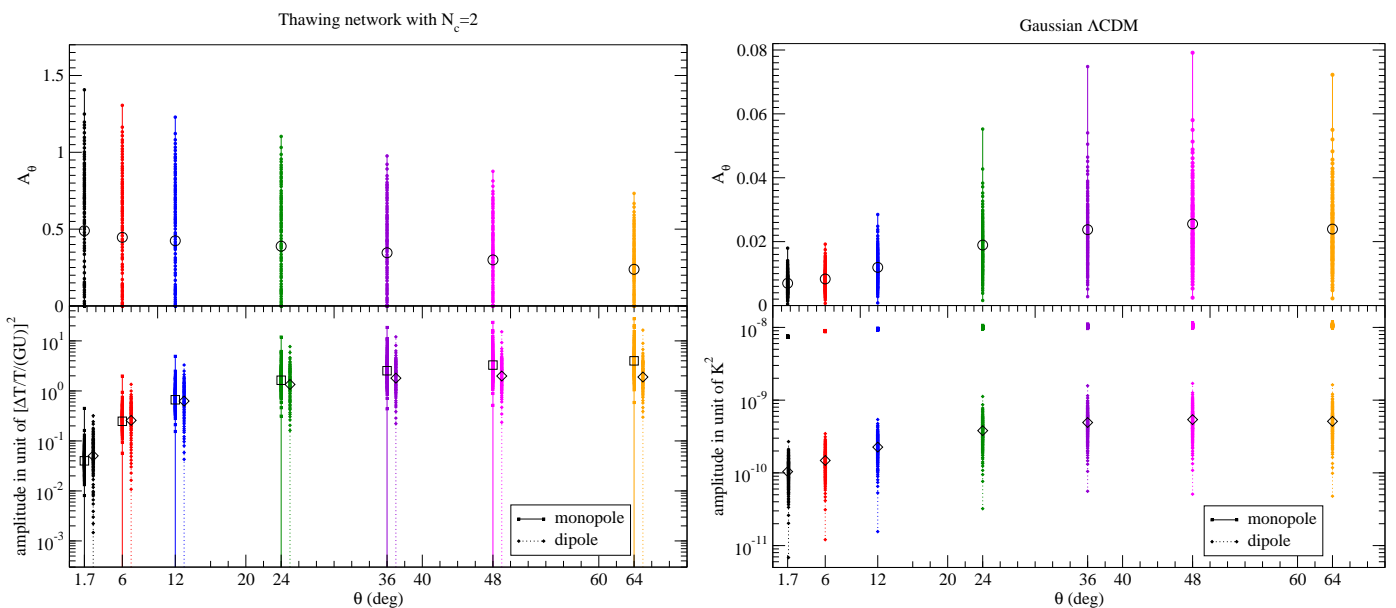

Figure 7. Dependency of the dipole modulation amplitude $A_{\theta}=\sigma_{\text {dip }}^{2} /\left(2 \sigma_{\text {mon }}^{2}\right)$ with respect to the averaging angle $\theta$ used to construct the local variance maps. The left panels are for thawing strings and can be compared to the expected pure Gaussian $\Lambda$ CDM realizations plotted on the right panels. Each point at a given $\theta$ represents one of the 1024 realizations while the largest black circle is the mean value. The lower panels show the absolute value of the monopole $\left(\sigma_{\text {mon }}^{2}\right)$ and dipole $\left(\sigma_{\text {dip }}^{2}\right)$ values (the string dipole values have been slightly shifted to the right for clarity).

\begin{tabular}{|c|c|c|c|c|c|c|c|}
\hline$\theta$ & $1.7^{\circ}$ & $6^{\circ}$ & $12^{\circ}$ & $24^{\circ}$ & $36^{\circ}$ & $48^{\circ}$ & $64^{\circ}$ \\
\hline$\left\langle A_{\theta}\right\rangle_{\text {thaw }}$ & 0.49 & 0.45 & 0.42 & 0.39 & 0.35 & 0.30 & 0.24 \\
\hline$\left\langle\sigma_{\text {dip }}^{2}\right\rangle_{\text {thaw }} /(T G U)^{2}$ & 0.05 & 0.26 & 0.63 & 1.34 & 1.80 & 1.98 & 1.90 \\
\hline$\left\langle\sigma_{\text {mon }}^{2}\right\rangle_{\text {thaw }} /(T G U)^{2}$ & 0.04 & 0.25 & 0.67 & 1.63 & 2.53 & 3.28 & 3.99 \\
\hline
\end{tabular}

Table 1. Mean values of $A_{\theta}, \sigma_{\text {mon }}^{2}$ and $\sigma_{\text {dip }}^{2}$ obtained over 1024 realizations of the thawing string networks with $N_{\mathrm{c}}=2$ (see also figure 7 ).

variance maps averaged over circles of $6^{\circ}$. Because a dipole modulation may also appear within the ordinary temperature anisotropies of inflationary origin, figure 6 also shows the value of $\sigma_{\text {dip }}^{2} /\left(2 \sigma_{\text {mon }}^{2}\right)$ obtained from 1024 random realizations of a purely Gaussian $\Lambda$ CDM anisotropies (also convolved with a 10' Gaussian beam). These Gaussian CMB maps have been generated using the HEALPix library [63] from an angular power spectrum calculated by means of the CAMB code [66]. The cosmological parameter values have been set according to the Planck 2015 favoured values [67], assuming a negligible contribution from tensor modes. For the Gaussian $\Lambda \mathrm{CDM}$ realizations, one gets $\left\langle A_{6}\right\rangle_{\Lambda \mathrm{CDM}}=8.3 \times 10^{-3}$ whereas the strings with $N_{\mathrm{c}}=2$ gives $\left\langle A_{6}\right\rangle_{\text {thaw }}=0.45$. As can be seen in figure 6 , the value of $\left\langle A_{6}\right\rangle_{\text {thaw }}$ is not very representative of the actual distribution, this one being actually multivalued. There is a sharp peak at vanishing value of $A_{6}$ while there is another local maximum around $A_{6} \simeq 0.7$. As mentioned before, this is because, for $N_{\mathrm{c}}=2$, around one quarter of the thawing string realizations produces no string on our past light cone. Therefore, a more representative value of the dipole modulation generically generated when strings are actually present is $A_{6} \simeq 0.7$; which is more than two orders of magnitude greater than the Gaussian expected value. 


\begin{tabular}{|c|c|c|c|c|c|c|c|}
\hline$\theta$ & $1.7^{\circ}$ & $6^{\circ}$ & $12^{\circ}$ & $24^{\circ}$ & $36^{\circ}$ & $48^{\circ}$ & $64^{\circ}$ \\
\hline$\left\langle A_{\theta}\right\rangle_{\Lambda \mathrm{CDM}}$ & 0.007 & 0.008 & 0.012 & 0.019 & 0.024 & 0.025 & 0.024 \\
\hline $10^{-2}\left\langle\sigma_{\text {dip }}^{2}\right\rangle_{\Lambda \mathrm{CDM}}\left(\mu \mathrm{K}^{2}\right)$ & 1.04 & 1.48 & 2.26 & 3.80 & 4.93 & 5.39 & 5.11 \\
\hline $10^{-4}\left\langle\sigma_{\text {mon }}^{2}\right\rangle_{\Lambda \mathrm{CDM}}\left(\mu \mathrm{K}^{2}\right)$ & 0.75 & 0.89 & 0.95 & 1.00 & 1.03 & 1.05 & 1.06 \\
\hline
\end{tabular}

Table 2. Mean values of $A_{\theta}, \sigma_{\text {mon }}^{2}$ and $\sigma_{\text {dip }}^{2}$ obtained over 1024 realizations of Gaussian $\Lambda$ CDM maps (see also figure 7).

\subsection{Averaging angle dependency}

Figure 7 shows the dependency of $A_{\theta}$ with respect to the local variance map averaging angle $\theta$, for both the thawing strings scenario with $N_{\mathrm{c}}=2$ (left panel) and the pure $\Lambda$ CDM model (right panel). There is a weak dependence of $A_{\theta}$ with respect to $\theta$ for both the strings and $\Lambda \mathrm{CDM}$, with the notable difference that the distribution of $A_{\theta}$ slightly goes down for strings whereas it increases for $\Lambda \mathrm{CDM}$ for larger values of $\theta$. For essentially all angles, $A_{\theta}$ generated by strings is two orders of magnitude greater than the Gaussian one. Moreover, the distribution of $A_{\theta}$ has a large variance, over 1024 realizations the maximum value may be larger than unity while the non-vanishing value for the minimum may be as low as $\mathcal{O}(1) \times 10^{-3}$. For instance, for $\theta=6^{\circ}$, one gets $\min \left\{A_{6} \mid A_{6} \neq 0\right\}=4 \times 10^{-3}$. Let us notice that the values obtained here for $\Lambda \mathrm{CDM}$ (right panels) are close to the ones derived from the Planck Full Focal Plane isotropic simulations (FFP6) of Ref. [58]. This agreement might be surprising at first, as our simulations are pure Gaussian $\Lambda$ CDM without any instrumental noise, but, at these scales of interest, most of the variance is indeed generated by the CMB.

The lower panels of figure 7 display the absolute value of the fitted monopole $\sigma_{\text {mon }}^{2}$ and dipole $\sigma_{\text {dip }}^{2}$ with respect to $\theta$. As opposed to the $\Lambda$ CDM case, in which both of these values increase only by a small factor with $\theta$, the delayed scaling string variance maps exhibit an increase by almost two orders of magnitude from $\theta=1.7^{\circ}$ to $64^{\circ}$. Such an effect results from both the actual string induced temperature patterns together with the construction of the local variance map. As can be seen in figure 5, the number of sky directions which are sensitive to the presence of a localized temperature pattern obviously increases with the opening angle $\theta$. This effect does not change very much the amplitude (and direction) of the modulation but boosts the averaged value of both the monopole and dipole simultaneously. On the contrary, because Gaussian $\Lambda \mathrm{CDM}$ fluctuations generate the same variance over all directions, changing the opening angle $\theta$ does not fundamentally modify the mean value of the monopole and dipole.

Qualitatively, the previous results suggest that if a few thawing strings actually contribute to the CMB sky, in addition to the Gaussian $\Lambda$ CDM anisotropies, they can produce a dipole modulation in the local variance map. The lower panels of figure 7 show that a typical signature is that such a modulation should be detectable only on the large angular scales.

\subsection{Mixture of string-induced and Gaussian anisotropies}

\subsubsection{Mean values}

We now consider that a few strings actually contribute to the overall CMB anisotropies, i.e., $\delta T=\delta T_{\Lambda \text { CDM }}+\delta T_{\text {thaw }}$ in which both contributions are assumed to be uncorrelated ${ }^{2}$.

\footnotetext{
${ }^{2}$ Such an assumption may no longer be true in some particular scenarios [68].
} 

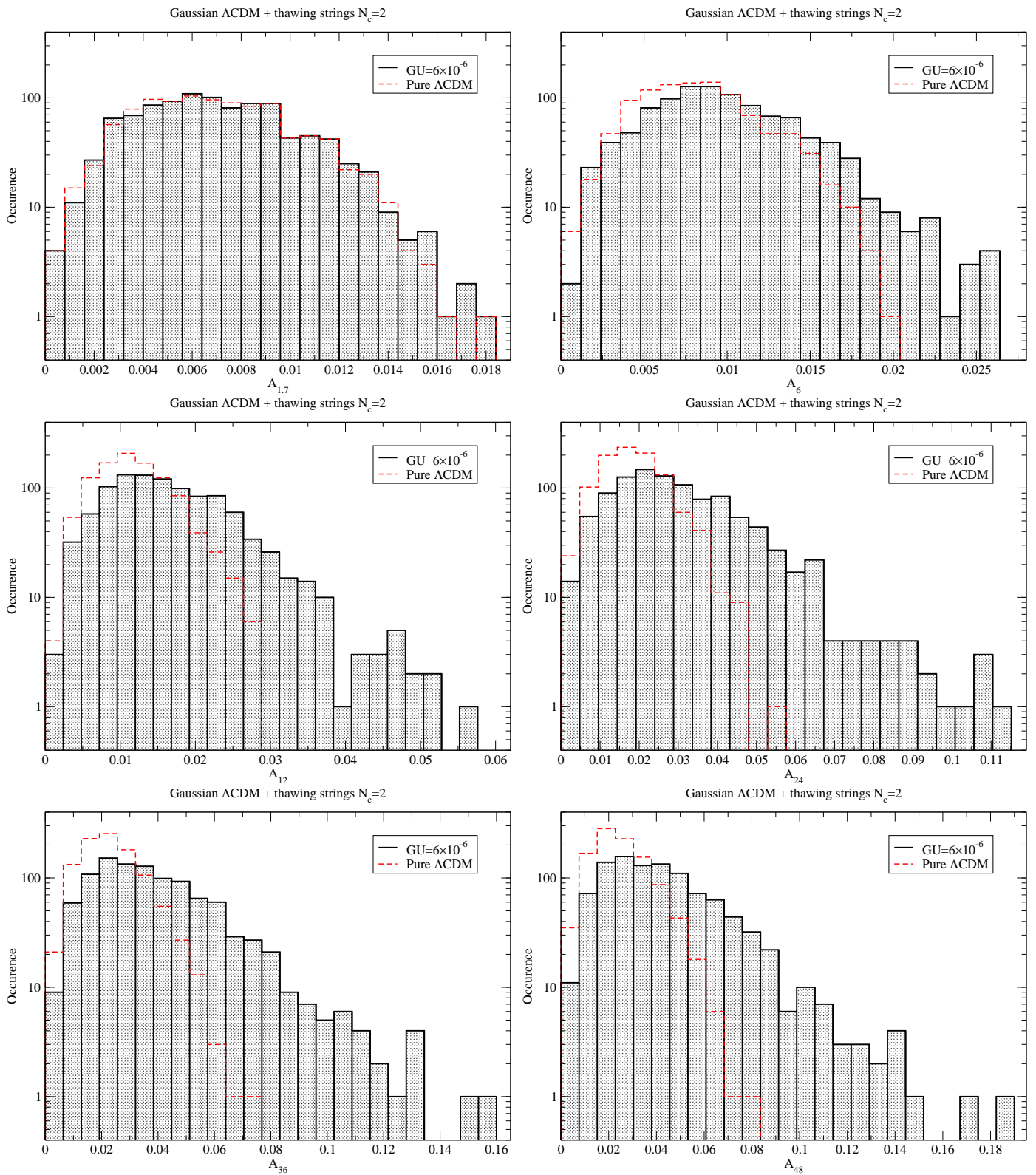

Figure 8. Distribution of the dipole modulation amplitude $A_{\theta}$ obtained for the opening angles $\theta=1.7^{\circ}, \theta=6^{\circ}, \theta=12^{\circ}, \theta=24^{\circ}, \theta=36^{\circ}$ and $\theta=48^{\circ}$ from a mixture of Gaussian $\Lambda \mathrm{CDM}$ fluctuations and thawing strings with $N_{\mathrm{c}}=2$ and $G U=6 \times 10^{-6}$. The pure Gaussian $\Lambda \mathrm{CDM}$ distributions have been reported for comparison. For angles $\theta \leq 2^{\circ}$, there are essentially no differences with respect to pure Gaussian anisotropies whereas significantly larger dipole amplitudes appear for $\theta \geq 6^{\circ}$.

Denoting by $\mathcal{M} \equiv \sigma_{\text {mon }}^{2}$ and $\mathcal{D} \equiv \sigma_{\text {dip }}^{2}$ one gets

$$
\begin{aligned}
A & =\frac{\mathcal{D}_{\Lambda \mathrm{CDM}}+\mathcal{D}_{\text {thaw }}}{2\left(\mathcal{M}_{\Lambda \mathrm{CDM}}+\mathcal{M}_{\text {thaw }}\right)} \simeq\left(1-\frac{\mathcal{M}_{\text {thaw }}}{\mathcal{M}_{\Lambda \mathrm{CDM}}}\right) A_{\Lambda \mathrm{CDM}}+\frac{\mathcal{M}_{\text {thaw }}}{\mathcal{M}_{\Lambda \mathrm{CDM}}} A_{\text {thaw }}, \\
& \simeq\left[1-\frac{\overline{\mathcal{M}}_{\text {thaw }}}{\overline{\mathcal{M}}_{\Lambda \mathrm{CDM}}}(G U)^{2}\right] A_{\Lambda \mathrm{CDM}}+\frac{\overline{\mathcal{M}}_{\text {thaw }}}{\overline{\mathcal{M}}_{\Lambda \mathrm{CDM}}}(G U)^{2} A_{\text {thaw }},
\end{aligned}
$$


where we have kept only the leading order terms in $\mathcal{M}_{\text {thaw }} / \mathcal{M}_{\Lambda \mathrm{CDM}}$ in the first line and introduced the dimensionless quantities

$$
\overline{\mathcal{M}}_{\text {thaw }} \equiv \frac{\mathcal{M}_{\text {thaw }}}{(T G U)^{2}}, \quad \overline{\mathcal{M}}_{\Lambda \mathrm{CDM}} \equiv \frac{\mathcal{M}_{\Lambda \mathrm{CDM}}}{T^{2}}
$$

The second line of Eq. (3.3) renders explicit the dependency in $G U$ and shows that the string contribution may boost the dipole modulation amplitude. For instance, matching a given dipole modulation amplitude $A_{\text {obs }}$, one obtains

$$
G U \simeq \sqrt{\frac{\overline{\mathcal{M}}_{\Lambda \mathrm{CDM}}}{\overline{\mathcal{M}}_{\mathrm{thaw}}}} \sqrt{\frac{A_{\mathrm{obs}}-A_{\Lambda \mathrm{CDM}}}{A_{\text {thaw }}-A_{\Lambda \mathrm{CDM}}}},
$$

an expression valid only for $A_{\text {obs }}>A_{\Lambda \mathrm{CDM}}$ and $A_{\text {thaw }}>A_{\Lambda \mathrm{CDM}}$, which is the situation we are interested in. Taking a fiducial value $A_{\text {obs }} \simeq 0.06$ compatible with the one reported by the Planck collaboration [44, 48], and blindly using mean values from tables 1 and 2, one gets $G U \simeq 7.7 \times 10^{-6}$ for $\theta=64^{\circ}, G U \simeq 7.7 \times 10^{-6}$ for $\theta=48^{\circ}, G U \simeq 6.6 \times 10^{-6}$ for $\theta=36^{\circ}, G U \simeq 9.5 \times 10^{-6}$ for $\theta=24^{\circ}, G U \simeq 1.5 \times 10^{-5}$ for $\theta=12^{\circ}, G U \simeq 2.4 \times 10^{-5}$ for $\theta=6^{\circ}$ and $G U \simeq 5.2 \times 10^{-5}$ for $\theta=1.7^{\circ}$. These figures confirm that, for a given value of $G U=\mathcal{O}(1) \times 10^{-6}$, the strings may generate a dipole modulation compatible with the one measured, but solely on the large angular scales. Interestingly, these values are quite natural for GUT scale cosmic strings and are of the same order of magnitude as the ones quoted in section 2.4. Therefore, if delayed scaling strings in the thawing regime are the underlying cause of the dipole modulation, the (very few) associated temperature discontinuities should be detectable in the CMB sky, provided they are not masked by foregrounds.

\subsubsection{Local variance maps}

The values of $G U$ quoted before have been estimated by using the mean values of table 1, which may not be representative of the actual distribution. As can be checked in figure 7, the mean value of $\left\langle A_{\theta}\right\rangle_{\text {thaw }}$ underestimates the actual distribution of non-vanishing realizations. This effect can be taken into account by directly mixing the string and $\Lambda$ CDM simulated CMB maps. In figure 8, we have represented the actual distribution of $A_{\theta}$ obtained over 1024 local variance maps created from a mixture of random Gaussian $\Lambda \mathrm{CDM}$ anisotropies with random thawing string configurations having $N_{\mathrm{c}}=2$ and $G U=6 \times 10^{-6}$. These distributions show that, for such a value of $G U$, there is no significant deviation with respect to the $\Lambda$ CDM Gaussian distribution at small angles, $\theta \leq 2^{\circ}$. On the contrary, already for $\theta \geq 6^{\circ}$, the distribution of possible dipole modulation amplitudes in the mixed scenario exhibits a long tail towards large values. For $\theta=12^{\circ}$, amplitude larger than 0.06 remains rare but far more probable than in the pure Gaussian case whereas for $\theta \geq 24^{\circ}$ they appear in 5\% of the 1024 realizations. These numbers accordingly increases with the value of $G U$.

\subsection{Discussion}

In this section, we have shown that the thawing string network with $N_{\mathrm{c}}=2$ genuinely generates a large scale dipole modulation in the local variance maps. The string tension required for matching the currently observed value is of the order $G U=\mathcal{O}(1) \times 10^{-6}$, which suggests that the temperature patterns generated by these very few strings may be detectable with dedicated searches. Another parameter which affects the signature of a delayed string network in the CMB is the correlation length $\xi /\left.\eta\right|_{\text {lss }}$. As discussed before, for the value 

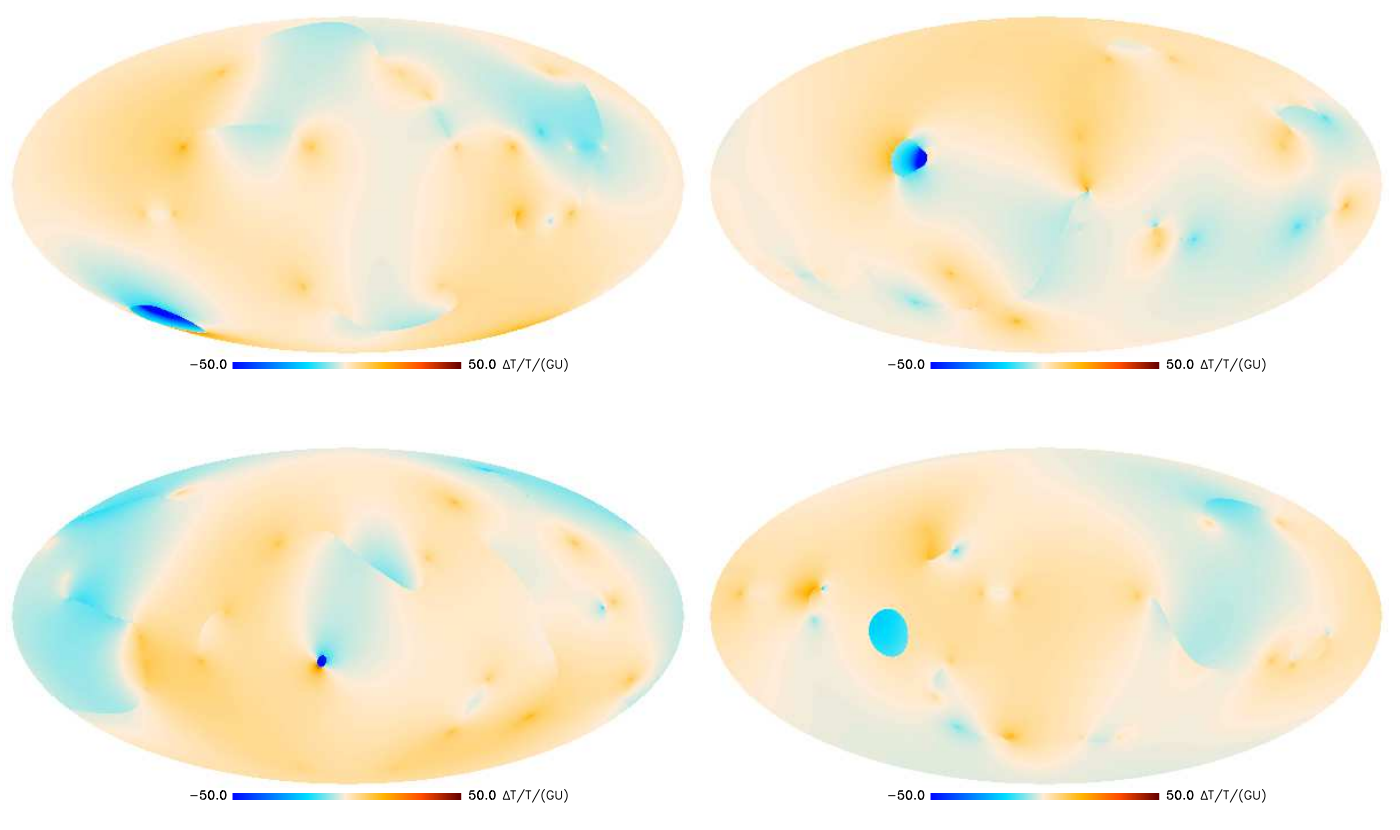

Figure 9. Four realizations of the CMB sky temperature generated by delayed scaling strings in the thawing regime and having an initial correlation length $\xi=16 \eta_{\mathrm{lss}}\left(N_{\mathrm{c}}=4\right)$. They exhibit one thawing loop generating a cold spot.

$\xi / \eta_{\text {lss }} \simeq 32$ used here $\left(N_{\mathrm{c}}=2\right)$, around a quarter of the realizations do not exhibit any strings on our past light cone and one may wonder what would be the situation with smaller initial correlation lengths. In the context of inflation, the strings would be formed later on.

\section{Cold spots from thawing loops}

Decreasing the correlation length of the string network at last scattering increases the number of strings on our past light cone and render the network properties closer to the delayed scaling scenario studied in Ref. [43]. As illustrated in figure 3 , for $N_{\mathrm{c}}=4$, the angular power spectrum is larger than for $N_{\mathrm{c}}=2$. At $\ell=10$, one has $C_{10}^{N_{\mathrm{c}}=4} / C_{10}^{N_{\mathrm{c}}=2} \simeq 4.6$ and at $\ell=400$, $C_{400}^{N_{\mathrm{c}}=4} / C_{400}^{N_{\mathrm{c}}=2} \simeq 6.0$. For values of $\xi$ smaller than the Hubble radius at last scattering, one would recover the configuration of a scaling network, having a large power spectrum and strongly constrained values of $G U$. Moreover, by the central limit theorem, one expects the properties of the dipole modulation to follow more closely a Gaussian statistics.

By decreasing $\xi$ from large super-Hubble values to Hubble-like values, one expects more thawing loops to be visible. Their typical behaviour is to shrink under their tension and their dynamics becomes close to the Nambu-Goto evolution in Minkowski space. The GKS effect on the CMB necessarily yields a cooler region inside the loop. Such a situation already occurs for $N_{\mathrm{c}}=2$ as can be seen in the first panel of figure 2 . If one increases $N_{\mathrm{c}}$, the angular size of the shrinking loops becomes smaller, their velocity higher and these events could provide a natural explanation of the so-called "cold spot" [69-72].

We have generated 1024 CMB maps associated with a thawing string network with $N_{\mathrm{c}}=4$ and thus having $\xi /\left.\eta\right|_{\text {lss }} \simeq 16$. A few maps have been represented in figure 9 and 

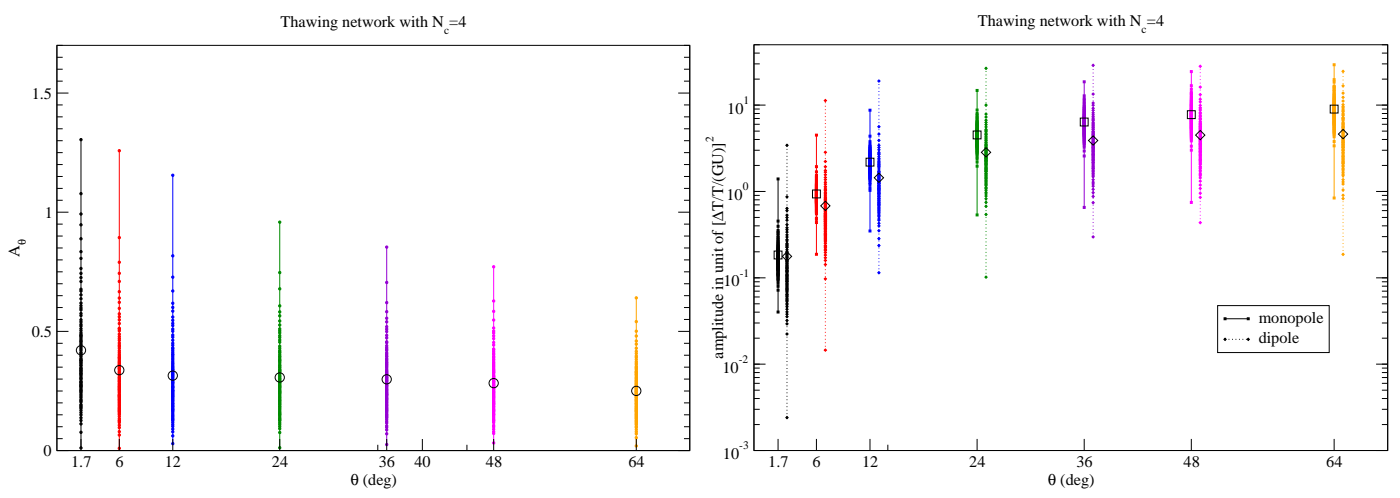

Figure 10. Modulation amplitude $A_{\theta}$, monopole $\sigma_{\text {mon }}^{2}$ and dipole $\sigma_{\text {dip }}^{2}$ in the local variance maps of thawing strings with $N_{\mathrm{c}}=4$ (to be compared with figure 7 ). The mean values are represented with a bigger symbol and have been reported in table 3 .

\begin{tabular}{|c|c|c|c|c|c|c|c|}
\hline$\theta$ & $1.7^{\circ}$ & $6^{\circ}$ & $12^{\circ}$ & $24^{\circ}$ & $36^{\circ}$ & $48^{\circ}$ & $64^{\circ}$ \\
\hline$\left\langle A_{\theta}\right\rangle_{\text {thaw }}$ & 0.42 & 0.34 & 0.31 & 0.31 & 0.30 & 0.28 & 0.25 \\
\hline$\left\langle\sigma_{\text {dip }}^{2}\right\rangle_{\text {thaw }} /(T G U)^{2}$ & 0.18 & 0.68 & 1.44 & 2.84 & 3.89 & 4.49 & 4.62 \\
\hline$\left\langle\sigma_{\text {mon }}^{2}\right\rangle_{\text {thaw }} /(T G U)^{2}$ & 0.18 & 0.93 & 2.19 & 4.51 & 6.37 & 7.75 & 8.99 \\
\hline
\end{tabular}

Table 3. Mean values of $A_{\theta}, \sigma_{\text {mon }}^{2}$ and $\sigma_{\text {dip }}^{2}$ obtained over 1024 realizations of the thawing string networks with $N_{\mathrm{c}}=4$.

show the presence of one thawing loop, at most. From these maps, we have derived the local variance maps averaged over various angles as in the previous section. The amplitude of the dipole modulation is still very large compared to the Gaussian $\Lambda \mathrm{CDM}$ value. The distribution and mean values of $A_{\theta}, \sigma_{\text {mon }}^{2}$ and $\sigma_{\text {dip }}^{2}$ for $N_{\mathrm{c}}=4$ have been plotted in figure 10 and reported in table 3. From Eq. (3.5), the typical value of $G U$ required to get $A_{\text {obs }} \simeq 0.06$ is $G U \simeq 5 \times 10^{-6}$ for $\theta=64^{\circ}, G U \simeq 5 \times 10^{-6}$ for $\theta=48^{\circ}, G U \simeq 5.3 \times 10^{-6}$ for $\theta=36^{\circ}$, $G U \simeq 6.5 \times 10^{-6}$ for $\theta=24^{\circ}, G U \simeq 9.7 \times 10^{-7}$ for $\theta=12^{\circ}, G U \simeq 1.4 \times 10^{-5}$ for $\theta=6^{\circ}$ and $G U \simeq 2.7 \times 10^{-5}$ for $\theta=1.7^{\circ}$. These numbers are slightly less than the ones obtained with $N_{\mathrm{c}}=2$ which may be attributed to the higher number of strings visible and the corresponding larger value of the mean variance (see table 3 ).

In order to discuss the actual distribution of a mixture of Gaussian $\Lambda$ CDM anisotropies with delayed scaling strings with $N_{\mathrm{c}}=4$, we have chosen a fiducial value of $G U=3 \times 10^{-6}$ (half of the value taken for $N_{\mathrm{c}}=2$ ). Along the same lines as in section 3 , the 1024 local variance maps of the mixture have been fitted with a dipole modulation to extract the amplitude $A_{\theta}$. The obtained distributions of $A_{\theta}$ are represented in figure 11. As for $N_{\mathrm{c}}=2$, the distributions remain very close to the $\Lambda$ CDM ones for angles $\theta \leq 2^{\circ}$ whereas they deviate significantly at larger opening angles. Comparing figures 8 and 11, one notices that, in spite of the reduced value of $G U$ used here, there are more outliers at large values of $A_{\theta}$ around intermediate angles. This is particularly visible in the distribution of $A_{6}\left(\theta=6^{\circ}\right)$ and $A_{12}$ $\left(\theta=12^{\circ}\right)$. In fact, all of the eleven local variance maps of figure 11 exhibiting $A_{6}>0.02$ contain a thawing loop producing one cold spot (four of them corresponds to the string configurations represented in figure 9). On the contrary, for angles $\theta \geq 24^{\circ}$, the distributions 

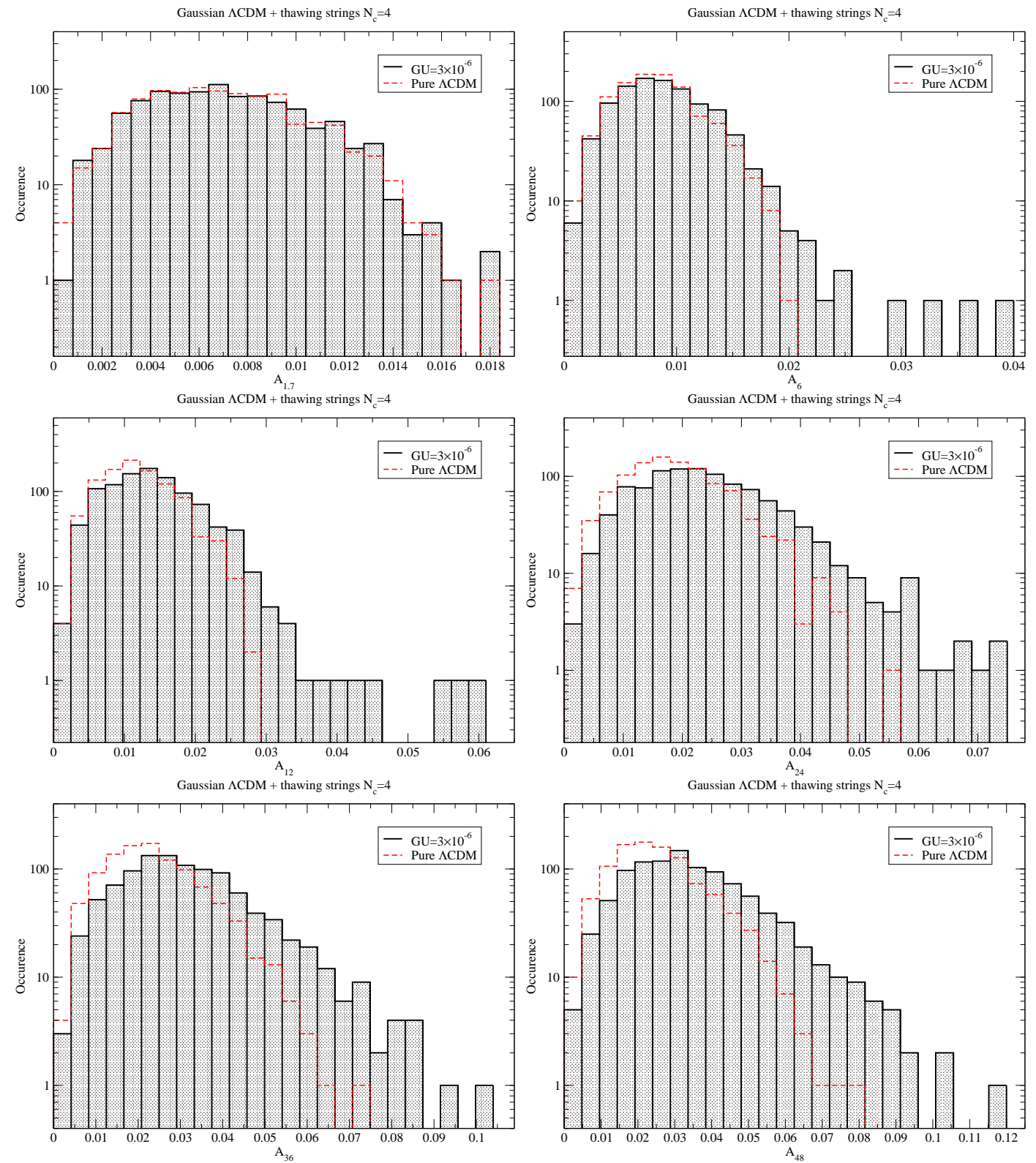

Figure 11. Distribution of the dipole modulation amplitude $A_{\theta}$ obtained for the opening angles $\theta=1.7^{\circ}, \theta=6^{\circ}, \theta=12^{\circ}, \theta=24^{\circ}, \theta=36^{\circ}$ and $\theta=48^{\circ}$ from a mixture of Gaussian $\Lambda \mathrm{CDM}$ fluctuations and thawing strings with $N_{\mathrm{c}}=4$ and $G U=3 \times 10^{-6}$. The pure Gaussian $\Lambda \mathrm{CDM}$ distributions have been reported for comparison.

of $A_{\theta}$ for $N_{\mathrm{c}}=4$ have a smaller tail towards large values than for $N_{\mathrm{c}}=2$. This is the expected behaviour due to both the smaller value of $G U$ and the larger number of strings on the past light cone. The latter effect is indeed rendering less probable the generation of an anisotropy looking like a pure dipole on the largest scales as the anisotropy patterns associated with the higher number of strings start to interfere. The boosting of the dipole modulation when a loop is present comes from the averaging effect associated with the local variance map making procedure, as illustrated in figure 5. The thawing loop, and its associated cold spot, 
induces a maximal effect when the averaging angle captures the typical angular size of the loop. For the situation discussed here, i.e., $N_{\mathrm{c}}=4$, this typically occurs for $\theta$ in the range $6^{\circ}-12^{\circ}$. Increasing $N_{\mathrm{c}}$ would decrease the angles at which such an effect takes place.

\section{Conclusion}

In this paper we have discussed the observational consequences of the delayed scaling string scenario in which cosmic strings, and possibly fundamental strings, are generated during inflation. If these objects are not diluted too much, namely, if they are produced in the last 60 e-folds of inflation, they may re-enter the Hubble radius in a recent past. Using NambuGoto numerical simulations in FLRW spacetime, we have studied the "thawing regime" in which strings crossing our past light cone decouple from the Hubble flow after recombination. Because visible strings are yet quasi-static and very rare, they can only distort the CMB in one or two directions in the sky while the generated patterns may be significantly different than line discontinuities. Constraints from the angular power spectrum and direct searches of temperature discontinuities typically allows values of the thawing string tension to be as large as a few times $10^{-6}$.

By running a thousand of Nambu-Goto cosmic string simulations, we have generated as many realizations of the CMB sky. We have shown that the thawing string induced signal mixed with pure Gaussian $\Lambda$ CDM anisotropies can mimic a large scale dipole modulation in the local variance maps. Both the string configurations having $\xi /\left.\eta\right|_{\text {lss }}=32$ and $\xi /\left.\eta\right|_{\text {lss }}=16$ can produce a dipole modulation of amplitude compatible with the observed value $A_{\text {obs }} \simeq 0.06$ provided their tension $G U=\mathcal{O}(1) \times 10^{-6}$. In addition, from the network configurations having $\xi /\left.\eta\right|_{\text {lss }}=16$, various realizations exhibit a shrinking loop on our past light cone whose generic property is to induce a cold spot in the CMB sky. In the local variance maps, this loop boosts the dipole modulation amplitude around angular scales close to the actual angular size of the loop. We have not discussed much smaller correlation length as we expect these situations to more closely follow the delayed scaling discussed in Ref. [43]. Indeed, reducing significantly more the correlation length $\xi$ at last scattering is expected to produce more loops of smaller angular sizes while increasing the overall number of visible strings thereby pushing the statistics closer to the Gaussian situation. At the same time, the amplitude of the angular power spectrum increases and the corresponding constraints on $G U$ would certainly prevent these strings to be visible at large angles. We have also not discussed the opposite situation in which $\xi /\left.\eta\right|_{\text {lss }} \gg 32$ because, already for $\xi /\left.\eta\right|_{\text {lss }} \simeq 32$, around a quarter of all realizations contain no string intercepting our past light cone. Therefore, larger correlation lengths at last scattering would correspond to a situation in which strings remain essentially invisible today. Finally, we left for a future work any attempt to extract the Bayesian evidence for a mixture of strings and $\Lambda \mathrm{CDM}$ fluctuations to explain both the large scale dipole modulation and the cold spot. It is indeed a non-trivial problem as determining any prior distribution, as for instance on the angular size and number of thawing loops, should rely on a significant larger number of Nambu-Goto string simulations.

Other discrimination tests could however be envisaged to examine further if thawing strings are the actual source of the observed large scale anomalies and the cold spot. For instance, because strings induce a genuinely non-Gaussian signal, local variance maps should correlate in a particular way with local skewness or local kurtosis maps and this could be used to disambiguate thawing strings from other sources. Moreover, the hypothesis that the cold spot is a shrinking loop may be tested by examining the lensing patterns of the CMB on its 
edge [73]. This could already be done by using for instance small scales CMB telescopes [74, 75]. Depending on its shape and velocity, a shrinking cosmic string loop is expected to induce a warmer $\mathrm{CMB}$ temperature in the adjacent regions located just outside the loop. Another possible direct detection could be achieved via gravitational lensing observations by measuring the spatial pattern on the deformation of photon path, which provides an evidence for the intervening matter distribution along the line of sight. All photons intercepting the loop should in addition be redshifted inside and in the same manner as the CMB. Finally, if the loop possesses kinks, or have enough dynamics to develop cusps, powerful bursts of gravitational waves could also be used as tracers $[76,77]$.

\section{Acknowledgments}

The work is supported in part by the Wallonia-Brussels Federation grant ARC 11/15-040, the Belgian Federal Office for Science, Technical and Cultural Affairs (C.R.), the Grand-in-Aid for Japan Society for the Promotion of Science (JSPS) Fellows No. 259800 (D.Y), and the JSPS Grand-in-Aid for Scientific Research No. $15 \mathrm{H} 02082$ (J.Y).

\section{References}

[1] D. Kirzhnits and A. Linde, Macroscopic consequences of the Weinberg model, Phys. Lett. B 42 (Dec., 1972) 471-474.

[2] T. W. B. Kibble, Topology of cosmic domains and strings., J. Phys. A 9 (1976) 1387-1398.

[3] E. Witten, Cosmic Superstrings, Phys. Lett. B153 (1985) 243.

[4] G. R. Dvali and S. H. H. Tye, Brane inflation, Phys. Lett. B450 (1999) 72-82, [hep-ph/9812483].

[5] M. B. Hindmarsh and T. W. B. Kibble, Cosmic strings, Rept. Prog. Phys. 58 (1995) 477-562, [hep-ph/9411342].

[6] R. Durrer, M. Kunz and A. Melchiorri, Cosmic structure formation with topological defects, Phys. Rep. 364 (June, 2002) 1-81, [astro-ph/0110348].

[7] J. Polchinski, Introduction to cosmic F- and D-strings, hep-th/0412244.

[8] A.-C. Davis, P. Brax and C. van de Bruck, Brane Inflation and Defect Formation, Phil. Trans. Roy. Soc. Lond. A366 (2008) 2833-2842, [0803.0424].

[9] E. J. Copeland and T. W. B. Kibble, Cosmic Strings and Superstrings, Proc. Roy. Soc. Lond. A466 (2010) 623-657, [0911.1345].

[10] M. Sakellariadou, Cosmic Strings and Cosmic Superstrings, Nucl. Phys. Proc. Suppl. 192-193 (2009) 68-90, [0902.0569].

[11] C. Ringeval, Cosmic strings and their induced non-Gaussianities in the cosmic microwave background, Adv. Astron. 2010 (2010) 380507, [1005.4842].

[12] C. Contaldi, M. Hindmarsh and J. Magueijo, The Power spectra of CMB and density fluctuations seeded by local cosmic strings, Phys.Rev.Lett. 82 (1999) 679-682, [astro-ph/9808201].

[13] M. Hindmarsh, C. Ringeval and T. Suyama, The CMB temperature bispectrum induced by cosmic strings, Phys. Rev. D80 (2009) 083501, [0908.0432].

[14] M. Hindmarsh, C. Ringeval and T. Suyama, The CMB temperature trispectrum of cosmic strings, Phys. Rev. D81 (2010) 063505, [0911.1241]. 
[15] D. Regan and M. Hindmarsh, The bispectrum of cosmic string temperature fluctuations including recombination effects, JCAP 1510 (2015) 030, [1508.02231].

[16] J. Urrestilla, N. Bevis, M. Hindmarsh and M. Kunz, Cosmic string parameter constraints and model analysis using small scale Cosmic Microwave Background data, JCAP 1112 (2011) 021, [1108.2730].

[17] Planck collaboration, P. Ade et al., Planck 2013 results. XXV. Searches for cosmic strings and other topological defects, Astron.Astrophys. 571 (2014) A25, [1303.5085].

[18] J. Lizarraga, J. Urrestilla, D. Daverio, M. Hindmarsh, M. Kunz et al., Constraining topological defects with temperature and polarization anisotropies, Phys.Rev. D90 (2014) 103504, [1408.4126].

[19] A. Lazanu, E. Shellard and M. Landriau, CMB power spectrum of Nambu-Goto cosmic strings, Phys. Rev. D91 (2015) 083519, [1410.4860].

[20] A. Lazanu and P. Shellard, Constraints on the Nambu-Goto cosmic string contribution to the $C M B$ power spectrum in light of new temperature and polarisation data, JCAP 1502 (2015) 024, [1410.5046].

[21] A. Albrecht and N. Turok, Evolution of cosmic string networks, Phys. Rev. D40 (Aug., 1989) 973-1001.

[22] D. P. Bennett and F. R. Bouchet, Cosmic-string evolution, Phys. Rev. Lett. 63 (Dec., 1989) 2776-2779.

[23] B. Allen and P. Shellard, Cosmic-string evolution - A numerical simulation, Phys. Rev. Lett. 64 (Jan., 1990) 119-122.

[24] D. P. Bennett and F. R. Bouchet, High-resolution simulations of cosmic-string evolution. I. Network evolution, Phys. Rev. D41 (Apr., 1990) 2408-2433.

[25] V. Vanchurin, K. Olum and A. Vilenkin, Cosmic string scaling in flat space, Phys. Rev. D72 (2005) 063514, [gr-qc/0501040].

[26] C. Ringeval, M. Sakellariadou and F. Bouchet, Cosmological evolution of cosmic string loops, JCAP 0702 (2007) 023, [astro-ph/0511646].

[27] L. Lorenz, C. Ringeval and M. Sakellariadou, Cosmic string loop distribution on all length scales and at any redshift, JCAP 1010 (2010) 003, [1006.0931].

[28] J. J. Blanco-Pillado, K. D. Olum and B. Shlaer, The number of cosmic string loops, Phys.Rev. D89 (2014) 023512, [1309.6637].

[29] P. Peter and C. Ringeval, A Boltzmann treatment for the vorton excess problem, JCAP 1305 (2013) 005, [1302.0953].

[30] R. Jeannerot, J. Rocher and M. Sakellariadou, How generic is cosmic string formation in SUSY GUTs, Phys. Rev. D68 (2003) 103514, [hep-ph/0308134].

[31] J. Rocher and M. Sakellariadou, D-term inflation, cosmic strings, and consistency with cosmic microwave background measurement, Phys. Rev. Lett. 94 (2005) 011303, [hep-ph/0412143].

[32] G. Lazarides and Q. Shafi, Extended Structures at Intermediate Scales in an Inflationary Cosmology, Phys.Lett. B148 (1984) 35.

[33] Q. Shafi and A. Vilenkin, Spontaneously Broken Global Symmetries and Cosmology, Phys.Rev. D29 (1984) 1870.

[34] E. T. Vishniac, K. A. Olive and D. Seckel, Cosmic Strings and Inflation, Nucl.Phys. B289 (1987) 717.

[35] L. Kofman and A. D. Linde, Generation of Density Perturbations in the Inflationary Cosmology, Nucl.Phys. B282 (1987) 555. 
[36] J. Yokoyama, Natural Way Out of the Conflict Between Cosmic Strings and Inflation, Phys.Lett. B212 (1988) 273.

[37] J. Yokoyama, INFLATION CAN SAVE COSMIC STRINGS, Phys. Rev. Lett. 63 (1989) 712.

[38] M. Nagasawa and J. Yokoyama, Phase transitions triggered by quantum fluctuations in the inflationary universe, Nucl.Phys. B370 (1992) 472-490.

[39] R. Basu and A. Vilenkin, Evolution of topological defects during inflation, Phys.Rev. D50 (1994) 7150-7153, [gr-qc/9402040].

[40] K. Freese, T. Gherghetta and H. Umeda, Moduli inflation with large scale structure produced by topological defects, Phys.Rev. D54 (1996) 6083-6087, [hep-ph/9512211].

[41] K. Kamada, Y. Miyamoto and J. Yokoyama, Evading the pulsar constraints on the cosmic string tension in supergravity inflation, JCAP 1210 (2012) 023, [1204.3237].

[42] J. Zhang, J. J. Blanco-Pillado, J. Garriga and A. Vilenkin, Topological Defects from the Multiverse, JCAP 1505 (2015) 059, [1501.05397].

[43] K. Kamada, Y. Miyamoto, D. Yamauchi and J. Yokoyama, Effects of cosmic strings with delayed scaling on CMB anisotropy, Phys.Rev. D90 (2014) 083502, [1407.2951].

[44] Planck collaboration, P. Ade et al., Planck 2013 results. XXIII. Isotropy and statistics of the CMB, Astron.Astrophys. 571 (2014) A23, [1303.5083].

[45] A. Notari, M. Quartin and R. Catena, CMB Aberration and Doppler Effects as a Source of Hemispherical Asymmetries, JCAP 1403 (2014) 019, [1304.3506].

[46] A. Rassat, J.-L. Starck, P. Paykari, F. Sureau and J. Bobin, Planck CMB Anomalies: Astrophysical and Cosmological Secondary Effects and the Curse of Masking, JCAP 1408 (2014) 006, [1405.1844].

[47] M. Quartin and A. Notari, On the significance of power asymmetries in Planck CMB data at all scales, JCAP 1501 (2015) 008, [1408.5792].

[48] Planck collaboration, P. Ade et al., Planck 2015 results. XVI. Isotropy and statistics of the $C M B, 1506.07135$.

[49] C. Ringeval and F. R. Bouchet, All Sky CMB Map from Cosmic Strings Integrated Sachs-Wolfe Effect, Phys.Rev. D86 (2012) 023513, [1204.5041].

[50] T. Vachaspati and A. Vilenkin, Formation and evolution of cosmic strings, Phys. Rev. D30 (Nov., 1984) 2036-2045.

[51] J. R. Gott III, Gravitational lensing effects of vacuum strings - Exact solutions, Astrophys. J. 288 (Jan., 1985) 422-427.

[52] N. Kaiser and A. Stebbins, Microwave anisotropy due to cosmic strings, Nature 310 (1984) 391-393.

[53] F. R. Bouchet, D. P. Bennett and A. Stebbins, Patterns of the cosmic microwave background from evolving string networks, Nature (London) 335 (Sept., 1988) 410-414.

[54] A. A. Fraisse, C. Ringeval, D. N. Spergel and F. R. Bouchet, Small-Angle CMB Temperature Anisotropies Induced by Cosmic Strings, Phys. Rev. D78 (2008) 043535, [0708.1162].

[55] R. J. Danos and R. H. Brandenberger, Canny Algorithm, Cosmic Strings and the Cosmic Microwave Background, Int.J.Mod.Phys. D19 (2010) 183-217, [0811.2004].

[56] R. J. Danos and R. H. Brandenberger, Searching for Signatures of Cosmic Superstrings in the CMB, JCAP 1002 (2010) 033, [0910.5722].

[57] E. Jeong, C. Baccigalupi and G. Smoot, Probing Cosmic Strings with Satellite CMB measurements, JCAP 1009 (2010) 018, [1004.1046]. 
[58] Y. Akrami, Y. Fantaye, A. Shafieloo, H. Eriksen, F. Hansen et al., Power asymmetry in WMAP and Planck temperature sky maps as measured by a local variance estimator, Astrophys.J. 784 (2014) L42, [1402.0870].

[59] P. Avelino, C. Martins and E. Shellard, Effects of inflation on a cosmic string loop population, Phys.Rev. D76 (2007) 083510, [0710.2210].

[60] S. Veeraraghavan and A. Stebbins, Large-scale microwave anisotropy from gravitating seeds, Astrophys. J. Lett. 395 (Aug., 1992) L55-L58.

[61] M. Hindmarsh, Small scale microwave background fluctuations from cosmic strings, Astrophys. J. 431 (1994) 534-542, [astro-ph/9307040].

[62] A. Stebbins and S. Veeraraghavan, Beyond the small-angle approximation for MBR anisotropy from seeds, Phys. Rev. D51 (Feb., 1995) 1465-1478, [astro-ph/9406067].

[63] K. Gorski, E. Hivon, A. Banday, B. Wandelt, F. Hansen et al., HEALPix - A Framework for high resolution discretization, and fast analysis of data distributed on the sphere, Astrophys.J. 622 (2005) 759-771, [astro-ph/0409513].

[64] E. Jeong and G. F. Smoot, The Validity of the Cosmic String Pattern Search with the Cosmic Microwave Background, Astrophys. J. Lett. 661 (May, 2007) L1-L4, [astro-ph/0612706].

[65] C. Gordon, W. Hu, D. Huterer and T. M. Crawford, Spontaneous isotropy breaking: a mechanism for cmb multipole alignments, Phys.Rev. D72 (2005) 103002, [astro-ph/0509301].

[66] A. Lewis, A. Challinor and A. Lasenby, Efficient computation of CMB anisotropies in closed FRW models, Astrophys.J. 538 (2000) 473-476, [astro-ph/9911177].

[67] Planck collaboration, R. Adam et al., Planck 2015 results. I. Overview of products and scientific results, 1502.01582 .

[68] C.-Y. Tseng and M. B. Wise, Inflaton Two-Point Correlation in the Presence of a Cosmic String, Phys.Rev. D80 (2009) 103512, [0908.0543].

[69] M. Cruz, M. Tucci, E. Martinez-Gonzalez and P. Vielva, The non-gaussian cold spot in wmap: significance, morphology and foreground contribution, Mon.Not.Roy.Astron.Soc. 369 (2006) 57-67, [astro-ph/0601427].

[70] S. Das and D. N. Spergel, CMB Lensing and the WMAP Cold Spot, Phys.Rev. D79 (2009) 043007, [0809.4704].

[71] M. Cruz, E. Martinez-Gonzalez, P. Vielva, J. Diego, M. Hobson et al., The CMB cold spot: texture, cluster or void?, Mon.Not.Roy.Astron.Soc. 390 (2008) 913, [0804.2904].

[72] F. Finelli, J. Garcia-Bellido, A. Kovacs, F. Paci and I. Szapudi, A Supervoid Imprinting the Cold Spot in the Cosmic Microwave Background, 1405.1555.

[73] F. Bernardeau and J.-P. Uzan, Cosmic string lens phenomenology: Model of Poisson energy distribution, Phys. Rev. D63 (2001) 023005, [astro-ph/0004102].

[74] S. Das, T. Louis, M. R. Nolta, G. E. Addison, E. S. Battistelli et al., The Atacama Cosmology Telescope: temperature and gravitational lensing power spectrum measurements from three seasons of data, JCAP 1404 (2014) 014, [1301.1037].

[75] SPT collaboration, K. T. Story et al., A Measurement of the Cosmic Microwave Background Gravitational Lensing Potential from 100 Square Degrees of SPTpol Data, Astrophys. J. 810 (2015) 50, [1412.4760].

[76] T. Damour and A. Vilenkin, Gravitational wave bursts from cusps and kinks on cosmic strings, Phys. Rev. D64 (2001) 064008, [gr-qc/0104026].

[77] S. Olmez, V. Mandic and X. Siemens, Gravitational-Wave Stochastic Background from Kinks and Cusps on Cosmic Strings, Phys.Rev. D81 (2010) 104028, [1004.0890]. 